\title{
Opportunity Loss Minimization and Newsvendor Behavior
}

\author{
Xinsheng Xu, ${ }^{1}$ Hong Yan, ${ }^{2}$ and Chi Kin $\mathrm{Chan}^{3}$ \\ ${ }^{1}$ Department of Mathematics, Binzhou University, Binzhou, China \\ ${ }^{2}$ Department of Logistics and Maritime Studies, The Hong Kong Polytechnic University, Kowloon, Hong Kong \\ ${ }^{3}$ Department of Applied Mathematics, The Hong Kong Polytechnic University, Kowloon, Hong Kong \\ Correspondence should be addressed to Xinsheng Xu; xxs0905@163.com
}

Received 11 January 2017; Revised 25 February 2017; Accepted 21 March 2017; Published 13 April 2017

Academic Editor: Jean Jacques Loiseau

Copyright (C) 2017 Xinsheng Xu et al. This is an open access article distributed under the Creative Commons Attribution License, which permits unrestricted use, distribution, and reproduction in any medium, provided the original work is properly cited.

\begin{abstract}
To study the decision bias in newsvendor behavior, this paper introduces an opportunity loss minimization criterion into the newsvendor model with backordering. We apply the Conditional Value-at-Risk (CVaR) measure to hedge against the potential risks from newsvendor's order decision. We obtain the optimal order quantities for a newsvendor to minimize the expected opportunity loss and CVaR of opportunity loss. It is proven that the newsvendor's optimal order quantity is related to the density function of market demand when the newsvendor exhibits risk-averse preference, which is inconsistent with the results in Schweitzer and Cachon (2000). The numerical example shows that the optimal order quantity that minimizes CVaR of opportunity loss is bigger than expected profit maximization (EPM) order quantity for high-profit products and smaller than EPM order quantity for lowprofit products, which is different from the experimental results in Schweitzer and Cachon (2000). A sensitivity analysis of changing the operation parameters of the two optimal order quantities is discussed. Our results confirm that high return implies high risk, while low risk comes with low return. Based on the results, some managerial insights are suggested for the risk management of the newsvendor model with backordering.
\end{abstract}

\section{Introduction}

The newsvendor model is a main research topic in inventory management, which has been applied to various settings including production planning and yield management. Since some basic assumptions of the classical newsvendor model are no longer suitable to meet the actual needs in practice, it is not surprising to see that many extensions of the classical newsvendor model have been suggested [1-3].

In the classical newsvendor model, it is assumed that all excess demands in a stockout situation are lost. In reality, it is often seen that all or part of the unsatisfied customers can wait for the demands to be replenished by the newsvendor soon afterwards, called backorder. For example, Corsten and Gruen [4] showed that about $15 \%$ of the unsatisfied customers are willing to accept the backorder invitations from the retailer in a stockout situation. In addition, with the improvement of customer service level, backlogging the excess demands in a stockout situation becomes more and more common in the real world. Therefore many papers addressing backordering in the newsvendor model have appeared in the operations management scope recently. Montgomery et al. [5] first introduced an exact solution procedure to determine the optimal policy of a newsvendor model with fixed partial backorder. Weng [6] considered a backorder case in which all the excess demand is backlogged but some of the excess demand may not be satisfied. San José et al. [7] introduced an inventory model with partial backlogging, where the unsatisfied demand is partially backlogged according to an exponential function and developed a general approach for finding the optimal policy to this model. Lodree [8] studied a two-level supply chain within a newsvendor framework which combines backorders and lost sales and also allows the retailer to start emergency replenishment. Lodree et al. [9] investigated the case in which all the excess demands are backlogged through an emergency procurement process, where it is assumed that costs incurred during the emergency procurement process include a variable ordering cost and a customer waiting cost. Zhou and Wang [10] extended the model proposed by 
Weng [6] to the case where the excess demand is partially backlogged and showed that the decentralized system would perform best if the manufacturer covers utterly the second production setup cost, which is opposite to that obtained in Weng [6]. Pando et al. [11] investigated the newsvendor model, where the backorders can be filled by an emergency lot. The optimal lot size and the maximum expected profit for such a case are obtained, and a general sensitivity analysis of the optimal policy with respect to the backorder rate and the parameters of the inventory system is also developed. Chen et al. [12] studied a compensation mechanism design problem with customer-choice behavior in a continuous review setting, where the production and demand processes are stochastic. Liu et al. [13] proposed an innovative twostep approach proposed based on an idea similar to the certainty equivalence principle and backordering is considered. L.-F. Hsu and J.-T. Hsu [14] developed economic production quantity (EPQ) models to determine the optimal production lot size and backorder quantity for a manufacturer under an imperfect production process. Braglia et al. [15] considered the continuous review $(r, q)$ inventory policy with complete backordering and developed a closed-form near-optimal solution. Khalilpourazari et al. [16] studied a multiproduct economic production quantity model with several real-world technical and physical constraints, where backordering is considered. Taleizadeh et al. [17] developed an inventory control model when replenishment intervals are probabilistic and partial backordering happens. Khalilpourazari and Pasandideh [18] studied a multi-item multiconstrained Economic Order Quantity model with nonlinear unit holding cost and partial backordering. It appears that the above papers mainly aim at maximizing the profit of a newsvendor by backlogging the unsatisfied demands or coordinating the optimal decisions of the supplier and the newsvendor in a two-echelon newsvendor model. However, little attention has been paid to the risk control for such a problem.

As stated above, the conventional approach to analyze the newsvendor model is based on the expected profit maximization (EPM), which considers that a newsvendor selects an order quantity to maximize his expected profit. The EPM order quantity is well documented in the newsvendor literature. However, studies found that the realized order quantity of the retailer in reality always deviates from EPM order quantity, which is referred to as "decision bias" in the newsvendor model $[19,20]$. Therefore studies are focused on behavior of newsvendor decisions and the "Pull to Center" (PTC) bias in the newsvendor model was proposed [19, 21, 22]. In Schweitzer and Cachon [19], the experimental results show that the newsvendor consistently orders less than EPM order quantity when EPM order quantity is larger than mean demand and orders more than EPM order quantity when EPM order quantity is lower than mean demand. In other words, the newsvendor always orders a quantity between EPM order quantity and mean demand, which is referred to as PTC bias in newsvendor literature. Then many papers are devoted to explaining such a newsvendor decision bias. For example, Ho et al. [23] proposed a behavioral theory by incorporating reference dependence to predict the newsvendor behavior and claimed that the newsvendor's actual orders exhibit PTC bias. Nagarajan and Shechter [24] clarified that the prospect theory cannot explain newsvendor's PTC bias. These authors showed numerically that prospect theory predicts overordering for high-profit products and underordering for low-profit products, which violates the PTC bias. Long and Nasiry [25] further stated that the prospect theory incorporating a reference point can explain PTC bias. However, many other researches do not support newsvendor's PTC bias. Lau et al. [26] reported that individual newsvendor behavior varies in practice and the PTC bias can be misleading in predicting individual newsvendor behavior. Based on the previous data in Schweitzer and Cachon [19] and Bolton and Katok [21], Lau et al. [26] conducted a new experiment and showed that the PTC bias does not adequately describe the individual newsvendor decision. Zhao and Geng [27] pointed out that the model in Long and Nasiry [25] is a special case of the general prospect theory model and showed that the general prospect theory model may be powerful in predicting the preferences of decision makers in inventory management. Y. Zhao and X. Zhao [28] found that a competing environment can cause participants to significantly increase their ordering levels in the highprofit group and increase their ordering oscillations in the low-profit group. In short, there are different opinions about PTC bias in the newsvendor model and relevant theoretical and experimental evidences are provided.

The experimental results in Schweitzer and Cachon [19] showed that subjects behave as if their utility function incorporates a preference to reduce the absolute difference between the chosen order quantity and realized demand. In light of this issue, this paper introduces the opportunity loss minimization criterion into the newsvendor model. The opportunity loss minimization criterion describes a decision maker's propensity to make a decision based on the need to minimize the opportunity loss after the decision has been made and/or after the outcome occurs. In other words, this criterion aims at obtaining a solution that minimizes the difference between the value of the selected solution and the optimal solution of the corresponding scenario. Up to now, the opportunity loss minimization criterion has been extensively studied and successfully applied to many settings in practice [29-32]. In this paper, we first obtain the optimal order quantity for a newsvendor to minimize expected opportunity loss. Further, to hedge against potential risks, we obtain the optimal order quantity for a newsvendor to minimize CVaR of opportunity loss. It is found that the optimal order quantity that minimizes CVaR of opportunity loss may be bigger or smaller than EPM order quantity, depending on the probability density function of market demand. The numerical results show that this optimal order quantity is bigger than EPM order quantity for high-profit products and smaller than EPM order quantity for lowprofit products when the market demand follows the normal distribution. In addition, it is shown that this optimal order quantity is bigger than EPM order quantity for both highprofit and low-profit products when market demand follows the exponential distribution. Recall that both the experiments in Schweitzer and Cachon [19] assumed that the market demand is uniformly distributed and the results are examined 
in such an environment. Our theoretical and numerical results show that the newsvendor's optimal order decision is related to the probability distribution of market demand when the newsvendor exhibits risk-averse preference.

The rest of this paper is organized as follows. Section 2 gives a detailed description of the proposed model and presents some preliminaries about the CVaR measure. Section 3 studies the optimal order quantities for a newsvendor that minimizes expected opportunity loss and CVaR of opportunity loss. Section 4 concludes the research.

\section{Model Description and Preliminaries}

In this section, a detailed description of the proposed model and some preliminaries about CVaR measure are presented.

2.1. Model Description. Consider a newsvendor model, where the market demand $\xi$ is a random variable with probability density function $f(\cdot)$ and cumulative distribution function $F(\cdot)$. Without loss of generality, assume that $F(0)=0$, $F(+\infty)=1$, and $F(\cdot)$ is continuously differentiable. For an order quantity $q$ and a realized market demand $D$, if $q \geq$ $D$, there exist some excess orders which can be salvaged; otherwise if $q<D$, there exist some excess demands from the unsatisfied customers. When $q<D$, it is assumed that all or part of the excess demands can be backlogged and the backorder rate (i.e., the percentage of the excess demands that can be backlogged) is denoted by $w(w \in[0,1])$. It is assumed that the backorders can be satisfied by the original supplier or some outsourcing inventories at a backorder price, which is higher than the wholesale price. Thus the newsvendor's realized profit can be given as

$$
\begin{aligned}
P(q)= & p \min \{q, D\}-c q+r(q-D)^{+} \\
& +w\left(p-c_{o}\right)(D-q)^{+},
\end{aligned}
$$

where $X^{+}=\max \{X, 0\}$. In (1), $p$ is the retail price, $c$ is the wholesale price, $r$ is the salvage price, and $c_{o}$ is the backorder price. Without loss of generality, it is assumed that $p>c_{o}>$ $c>r \geq 0$. In the right-hand side of (1), the first term denotes the sales income, the second term is the ordering cost, the third term is the salvage income from the excess orders, and the last term is the profit from backordering.

The newsvendor can attain his maximum profit when $q=$ $D$ and the maximum profit can be given as

$$
M(D)=(p-c) D \text {. }
$$

It follows from (1) and (2) that the opportunity loss of the newsvendor in choosing the order quantity $q$ is given as

$$
\begin{aligned}
& O(q)=M(D)-P(q) \\
& =(p-c) D-\left[p \min \{q, D\}-c q+r(q-D)^{+}\right. \\
& \left.\quad+w\left(p-c_{o}\right)(D-q)^{+}\right] .
\end{aligned}
$$

The opportunity loss $O(q)$ represents the absolute deviation of profit in choosing the order quantity $q$ from the realized market demand $D$. Recall that the experimental results of Schweitzer and Cachon [19] show that the subjects behave as if utility function incorporates a preference to reduce the absolute difference between the chosen quantity and realized demand. This paper therefore concentrates on selecting the optimal order quantity for the newsvendor to minimize the above opportunity loss.

2.2. The CVaR Measure. In an uncertain environment, let $l(x)$ be the decision maker's loss from a decision $x$. The VaR of $l(x)$ is defined as

$$
\operatorname{VaR}_{\alpha}[l(x)]=\inf \{y \in \mathbb{R} \mid \operatorname{Pr}\{l(x) \leq y\} \geq \alpha\},
$$

where $\operatorname{Pr}\{l(x) \leq y\}$ denotes the probability of $l(x)$ below the value of $y$ and $\alpha$ defines the confidence level. $\operatorname{VaR}_{\alpha}[l(x)]$ represents the decision maker's minimum loss under the confidence level $\alpha$. Based on VaR, Rockafellar and Uryasev $[33,34]$ introduced a CVaR measure. Taking $\operatorname{VaR}_{\alpha}[l(x)]$ as the targeted loss, the CVaR of $l(x)$ is defined as

$$
\mathrm{CVaR}_{\alpha}[l(x)]=E\left[l(x) \mid l(x) \geq \operatorname{VaR}_{\alpha}[l(x)]\right] .
$$

$\mathrm{CVaR}_{\alpha}[l(x)]$ represents the expected value of the loss exceeding the target level $\operatorname{VaR}_{\alpha}[l(x)]$. Hence, minimizing $\mathrm{CVaR}_{\alpha}[l(x)]$ is to find an optimal decision that minimizes the expectation of the loss above the target level. Therefore the CVaR measure provides a downside risk protection against possible losses for risk-averse decision makers. To compute, Rockafellar and Uryasev [33] introduced the auxiliary function,

$$
F(x, u)=u+\frac{1}{1-\alpha} E\left[(l(x)-u)^{+}\right],
$$

and proved that the optimal decision of minimizing $\mathrm{CVaR}_{\alpha}[l(x)]$ can be achieved by minimizing the function $F(x, u)$.

\section{Minimizing Opportunity Loss in Newsvendor Model}

In this section, we first obtain the optimal order quantity for a newsvendor that minimizes expected opportunity loss. Then we study the optimal order quantity for a newsvendor that minimizes CVaR of opportunity loss. All the proofs can be found in the Appendix.

Since the realized market demand cannot be observed before the selling season, the newsvendor cannot learn his realized opportunity loss from an order quantity. As a conventional approach, we first analyze the optimal order quantity for a newsvendor that minimizes expected opportunity loss $E[O(q)]$ ( $E$ is the expectation operator). We have the following result to address this issue.

Theorem 1. The optimal order quantity for a newsvendor that minimizes expected opportunity loss is given by

$$
q^{*}=F^{-1}\left[\frac{p-c-w\left(p-c_{o}\right)}{p-r-w\left(p-c_{o}\right)}\right] .
$$


It follows from (2) that the newsvendor's maximum profit $M(D)$ is independent of the order quantity $q$, thus minimizing the opportunity loss $O(q)$ equals maximizing the profit $P(q)$. Hence, the above expected opportunity loss minimization (EOLM) order quantity $q^{*}$ also equals the newsvendor's EPM order quantity.

It is known that the expected performance optimizing measure cannot guarantee that the realized performance falls within a small neighborhood of its expected value. In other words, the above EOLM criterion cannot effectively hedge against the potential risks, which may bring a great opportunity loss to the newsvendor. In recent years, more and more researchers have paid attention to risk management in inventory management and some efficient risk control criteria have been introduced. As a preferable downside risk control measure, the CVaR measure has been widely used in the newsvendor model and some interesting results have been obtained [35-42]. Following this line of research, we introduce the CVaR measure into this study and investigate the optimal order quantity for a newsvendor that minimizes CVaR of opportunity loss.

For the opportunity loss $O(q)$ of the newsvendor, we can define the $\operatorname{VaR}$ of $O(q)$ as

$$
\operatorname{VaR}_{\alpha}[O(q)]=\inf \{y \in R \mid \operatorname{Pr}\{O(q) \leq y\} \geq \alpha\} .
$$

It represents the minimum opportunity loss of the newsvendor under confidence level $\alpha$. Taking $\operatorname{VaR}_{\alpha}[O(q)]$ as the targeted opportunity loss, the newsvendor's CVaR of opportunity loss is given as

$$
\mathrm{CVaR}_{\alpha}[O(q)]=E\left[O(q) \mid O(q) \geq \operatorname{VaR}_{\alpha}[O(q)]\right] .
$$

This CVaR measure defines the expected value of opportunity loss exceeding target level $\operatorname{VaR}_{\alpha}[O(q)]$. Hence, it pays more attention to opportunity loss above target level $\operatorname{VaR}_{\alpha}[O(q)]$, while the opportunity loss below this target level is neglected. This is more appealing to the newsvendor, since the opportunity loss below target level clearly cannot be regarded as a risk to be hedged. Then we have the following result to minimize the CVaR of opportunity loss.

Theorem 2. The optimal order quantity $q^{\alpha}$ for a newsvendor that minimizes CVaR of opportunity loss is given as

$$
\begin{aligned}
q^{\alpha} & =\left((c-r) F^{-1}\left[\frac{(1-\alpha)\left(p-c-w\left(p-c_{o}\right)\right)}{p-r-w\left(p-c_{o}\right)}\right]\right. \\
& +\left(p-c-w\left(p-c_{o}\right)\right) \\
& \left.\cdot F^{-1}\left[\frac{(1-\alpha)\left(p-c-w\left(p-c_{o}\right)\right)}{p-r-w\left(p-c_{o}\right)}+\alpha\right]\right)(p-r \\
& \left.-w\left(p-c_{o}\right)\right)^{-1} .
\end{aligned}
$$

When $\alpha=0$, the risk-averse newsvendor turns to be risk-neutral. It follows with $\alpha=0$ in Theorem 2 that the optimal order quantity $q^{\alpha}$ reduces to EOLM (i.e., EPM) order quantity $q^{*}$ in Theorem 1 , which also is the EPM order quantity. The confidence level reflects the newsvendor's degree of risk aversion. The bigger the confidence level is, the more risk-averse the newsvendor becomes. Both Chen et al. [35] and Gotoh and Takano [36] showed that the optimal order quantity, for a newsvendor without backordering and maximizing $C V a R$ of profit, is decreasing in the confidence level and thus is smaller than EPM order quantity. In fact, we have proven that the corresponding optimal order quantity is given as

$$
q_{1}^{\alpha}=F^{-1}\left[\frac{(1-\alpha)\left(p-c-w\left(p-c_{o}\right)\right)}{p-r-w\left(p-c_{o}\right)}\right]
$$

Therefore $q_{1}^{\alpha}$ is decreasing in the confidence level and is smaller than EPM order quantity $q^{*}$ in Theorem 1 . The intuition for this result is clear: to maximize profit, potential risk comes only from possible excess orders. Therefore, a newsvendor that aims to maximize profit should order less to reduce the potential risks when he becomes more riskaverse. The more risk-averse the newsvendor is, the less he orders. However, the following result shows that this property does not hold when a newsvendor aims to minimize CVaR of opportunity loss.

Remark 3. The optimal order quantity $q^{\alpha}$ for a newsvendor that minimizes CVaR of opportunity loss may be increasing or decreasing in the confidence level $\alpha$. Recall that $q^{\alpha}$ reduces to EPM order quantity $q^{*}$ when $\alpha=0$. This remark implies that the optimal order quantity $q^{\alpha}$ may be more or less than EPM order quantity $q^{*}$. It follows with Theorem 1 that

$$
\begin{aligned}
& \frac{\partial q^{\alpha}}{\partial \alpha} \\
& \quad=\frac{(c-r)\left(p-c-w\left(p-c_{o}\right)\right)}{\left(p-r-w\left(p-c_{o}\right)\right)^{2} f(\theta) f(\kappa)}[f(\theta)-f(\kappa)],
\end{aligned}
$$

where

$$
\begin{aligned}
& \theta=F^{-1}\left[\frac{(1-\alpha)\left(p-c-w\left(p-c_{o}\right)\right)}{p-r-w\left(p-c_{o}\right)}\right], \\
& \kappa=F^{-1}\left[\frac{(1-\alpha)\left(p-c-w\left(p-c_{o}\right)\right)}{p-r-w\left(p-c_{o}\right)}+\alpha\right] .
\end{aligned}
$$

Therefore, the sign of $\partial q^{\alpha} / \partial \alpha$ is decided by the sign of $\Delta=$ $[f(\theta)-f(\kappa)]$, which may be positive or negative. If $f(\cdot)$ is increasing, we have $\Delta<0$ and $\partial q^{\alpha} / \partial \alpha<0$, since $\theta \leq \kappa$. Then $q^{\alpha}$ is decreasing in the confidence level and is smaller than EPM order quantity $q^{*}$. If $f(\cdot)$ is decreasing (e.g., the exponential distribution), we have $\Delta>0$ and $\partial q^{\alpha} / \partial \alpha>0$. Then $q^{\alpha}$ is increasing in the confidence level and is bigger than EPM order quantity $q^{*}$. If $f(\cdot)$ is a constant (e.g., the uniform distribution), we have $\Delta=0$ and $\partial q^{\alpha} / \partial \alpha=0$. Then $q^{\alpha}$ is the same as EPM order quantity $q^{*}$. Therefore, the change direction of optimal order quantity $q^{\alpha}$ with regard to confidence level is related to the probability density function of market demand. In the following, we give a numerical example on this conclusion. 

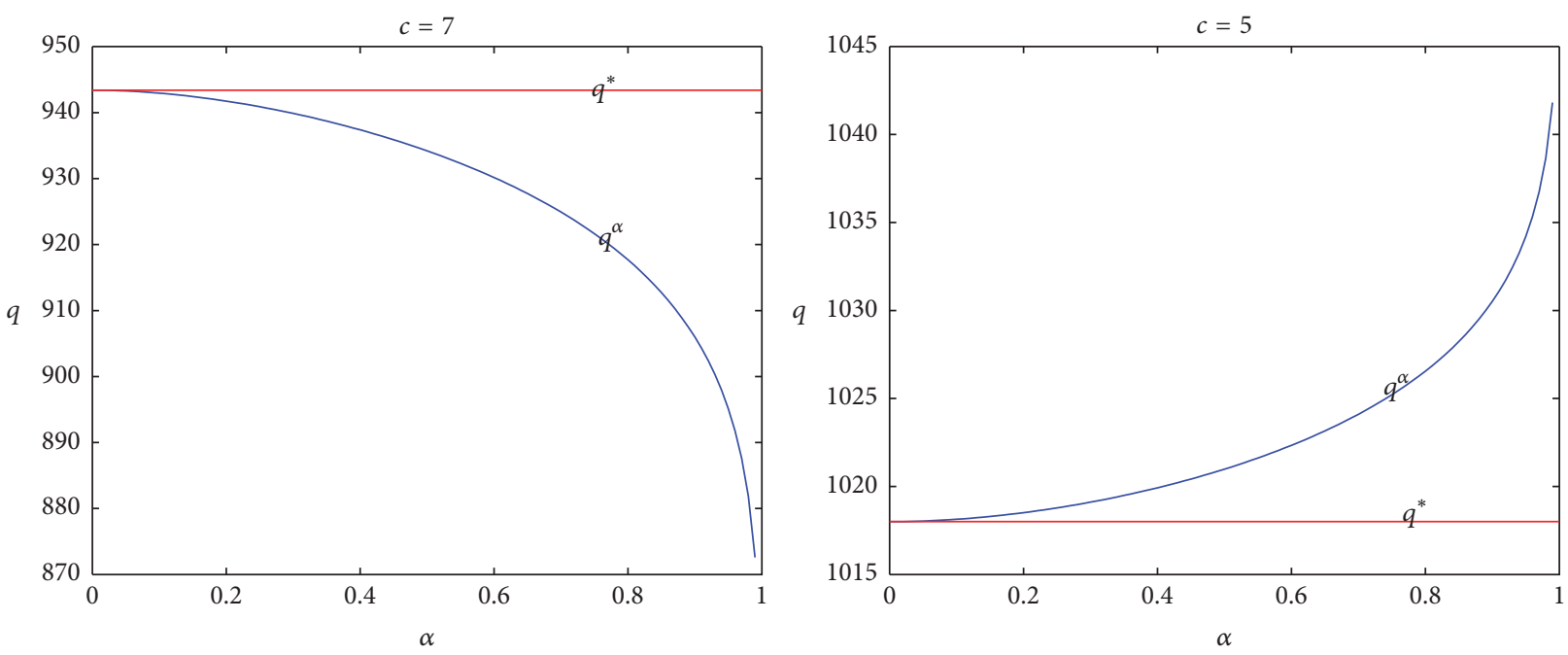

FIGURE 1: Optimal order quantity $q^{\alpha}$ for $c=7$ and $c=5$ when market demand follows the normal distribution $N\left(1000,100^{2}\right)$.
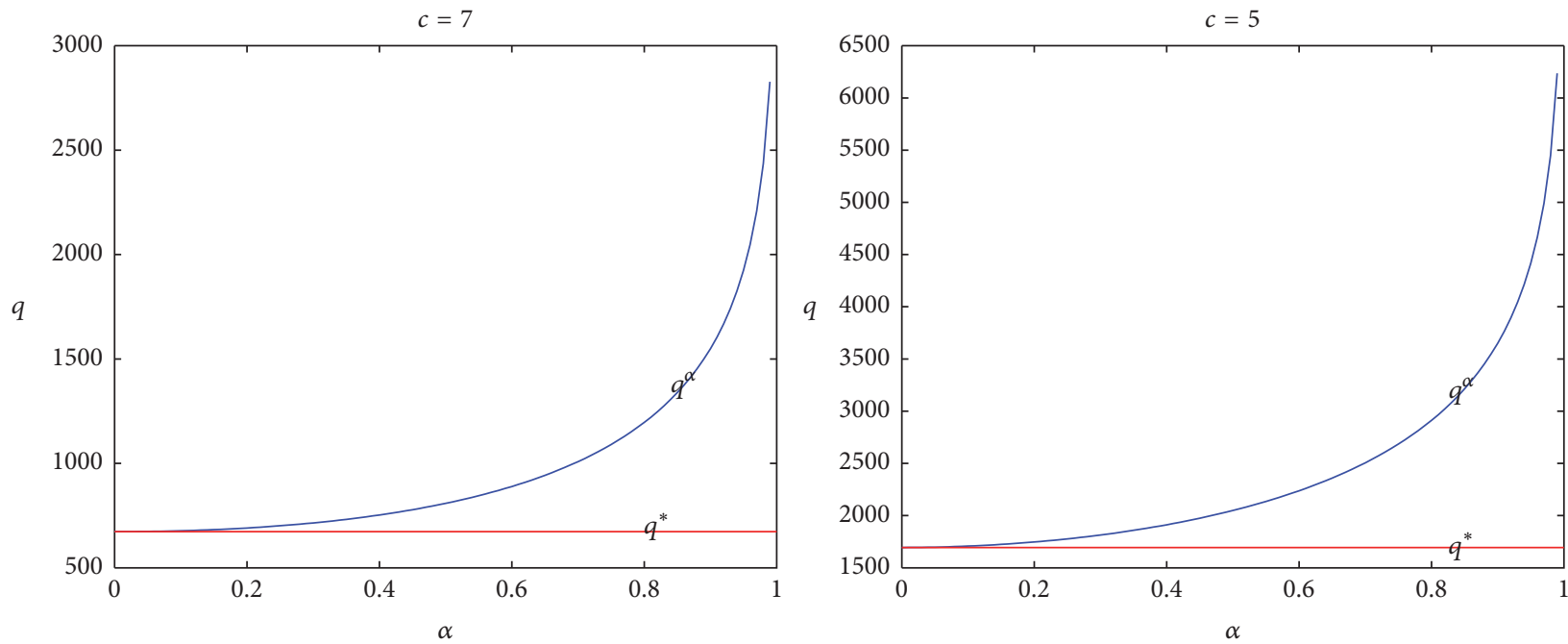

FIGURE 2: Optimal order quantity $q^{\alpha}$ or $c=7$ and $c=5$ when market demand follows the exponential distribution $E(0.0005)$.

Example 4. Consider the newsvendor model. Suppose that the market demand $\xi$ follows the normal distribution $N\left(1000,100^{2}\right)$ or exponential distribution $E(0.0005)$. We assume that the operations parameters are given as $p=10$, $r=2, c_{o}=8$, and $w=0.5$. For $c=7$ and $c=5$, we compute the newsvendor's optimal order quantities $q^{*}$ and $q^{\alpha}$ with different confidence level for the two market demand distributions. The results are given in Figures 1 and 2, respectively.

The newsvendor's understock loss and overstock loss in such a case are given as

$$
\begin{aligned}
& l_{u}=p-c-w\left(p-c_{o}\right), \\
& l_{o}=c-r .
\end{aligned}
$$

When understock loss is bigger than overstock loss, the product becomes the high-profit product defined in Schweitzer and Cachon $[19]^{1}$. Otherwise, the product becomes the lowprofit product defined in Schweitzer and Cachon [19].

In Figure 1, we plot the newsvendor's optimal order quantities $q^{*}$ and $q^{\alpha}$ when market demand follows the normal distribution $N\left(1000,100^{2}\right)$. In the first graph of Figure 1, $c=7$ and understock loss is smaller than overstock loss. It can be seen that the optimal order quantity $q^{\alpha}$ is decreasing with the confidence level and is smaller than EPM order quantity $q^{*}$. In the second graph of Figure $1, c=5$ and understock loss is higher than overstock loss. It is shown that the optimal order quantity $q^{\alpha}$ is increasing with the confidence level and is bigger than EPM order quantity $q^{*}$. These results imply that $q^{\alpha}$ is higher than EPM order quantity $q^{*}$ for highprofit products and smaller than EPM order quantity $q^{*}$ for 

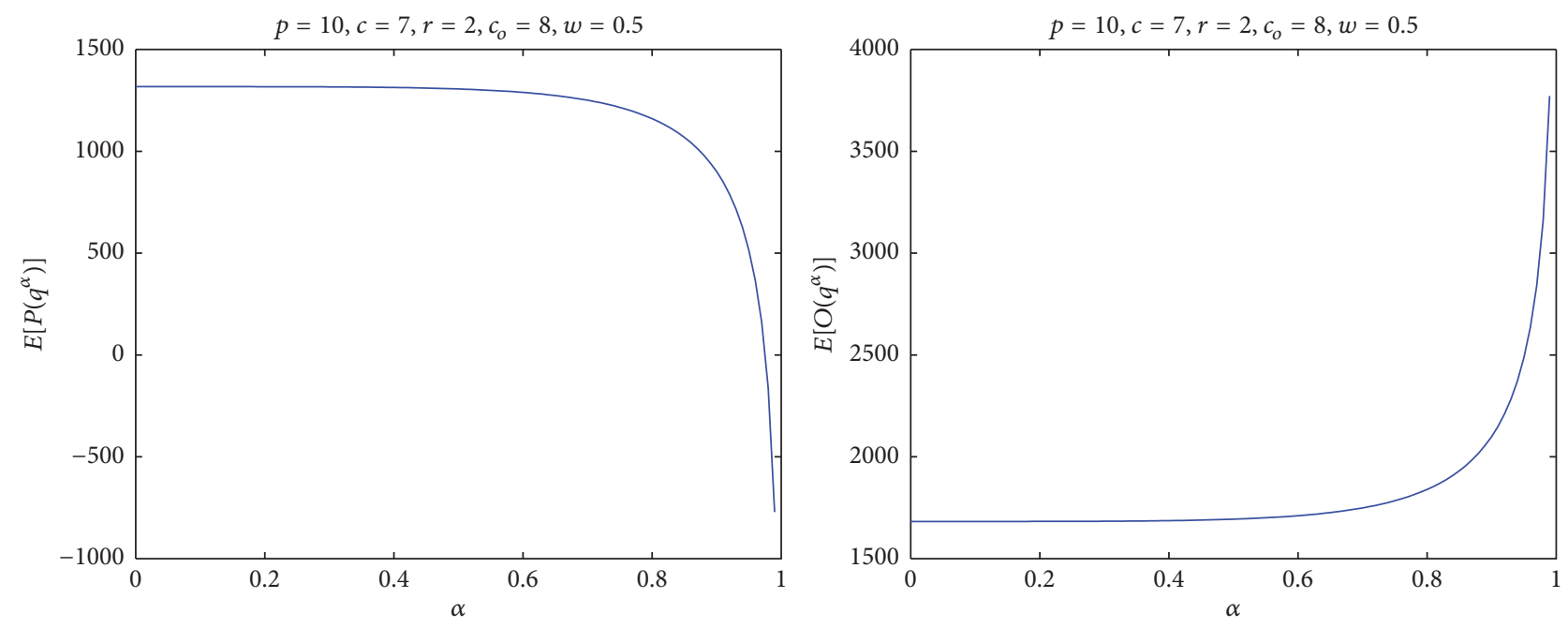

Figure 3: $E\left[P\left(q^{\alpha}\right)\right]$ and $E\left[O\left(q^{\alpha}\right)\right]$ with different confidence level $\alpha$.

low-profit products when market demand follows the normal distribution. This is consistent with the results in Eeckhoudt et al. [43] and in Nagarajan and Shechter [24]. However, this result is inconsistent with the experimental results in Schweitzer and Cachon [19].

In Figure 2, we plot the newsvendor's optimal order quantities $q^{*}$ and $q^{\alpha}$ when market demand follows the exponential distribution $E(0.0005)$. It is clear that the optimal order quantity $q^{\alpha}$ is increasing with the confidence level and is bigger than EPM order quantity $q^{*}$ for both high-profit and low-profit products.

These results imply that the newsvendor's optimal order decisions should consider the distribution of market demand and change with the product's market demand when the newsvendor exhibits risk-averse preference.

Remark 3 points out that the newsvendor that minimizes CVaR of opportunity loss may select a bigger or smaller order quantity so as to reduce potential risks when he becomes more risk-averse. Then how do the expected profit $E\left[P\left(q^{\alpha}\right)\right]$ and expected opportunity loss $E\left[O\left(q^{\alpha}\right)\right]$ under the optimal order quantity $q^{\alpha}$ change with the increase of the confidence level? We have the following results to address these two issues.

Theorem 5. The newsvendor's expected profit $E\left[P\left(q^{\alpha}\right)\right]$ under the optimal order quantity $q^{\alpha}$ is decreasing in the confidence level $\alpha$.

Theorem 6. The newsvendor's expected opportunity loss $E\left[O\left(q^{\alpha}\right)\right]$ under the optimal order quantity $q^{\alpha}$ is increasing in the confidence level $\alpha$.

The above results imply that the newsvendor will face a lower expected profit and a higher expected opportunity loss when he selects an order quantity with a bigger confidence level to reduce the potential risks. This confirms that high return follows with high risk and low risk comes with low return. We now give an example to show this result.
Example 7. Consider the newsvendor model. Suppose that the market demand $\xi$ follows the exponential distribution $E(0.001)$. The parameters are given as $p=10, c=7, r=2$, $c_{o}=8$, and $w=0.5$. With different confidence level, we plot the newsvendor's expected profit $E\left[P\left(q^{\alpha}\right)\right]$ and expected opportunity loss $E\left[O\left(q^{\alpha}\right)\right]$ under the optimal order quantity $q^{\alpha}$ in Figure 3.

Figure 3 shows that $E\left[P\left(q^{\alpha}\right)\right]$ is decreasing in the confidence level and $E\left[O\left(q^{\alpha}\right)\right]$ is increasing in the confidence level. This confirms the above results in Theorems 5 and 6 . So the newsvendor should strike a balance between risk control and opportunity loss minimization in selecting the optimal order quantity.

\section{Conclusions}

This paper revisits the newsvendor model. One objective is to find an order quantity that minimizes the absolute deviation of profit from the realized demand. We introduce a definition of opportunity loss and first obtain the optimal order quantity for a newsvendor that minimizes expected opportunity loss. To reduce the potential risks, we then introduce the CVaR measure to quantify the potential risks. Our paper thus contributes to the newsvendor literature in the following two aspects. First, we introduce the opportunity loss minimizing criterion into the decision framework of the newsvendor model with backordering and show how the optimal order quantities under this criterion interact with business conditions (e.g., the retail price and the backorder rate). This provides some managerial insights for the optimal decisions of the newsvendor in a backorder setting. For example, we find the following. (i) If the backorder rate increases, the newsvendor should order less to minimize opportunity loss. (ii) If the backorder price increases, the newsvendor should order more to minimize opportunity loss. Second, we integrate CVaR measure into the opportunity loss minimization criterion and obtain the optimal order 
quantity for the risk-averse newsvendor that minimizes CVaR of opportunity loss. The results show how the newsvendor's risk aversion influences his optimal order decision with backordering. (iii) A newsvendor may order more or less than EPM order quantity when the newsvendor exhibits riskaverse behavior, which depends on the probability density function of market demand. We provide numerical results to show that the newsvendor that minimizes CVaR of opportunity loss orders more than EPM order quantity for high-profit products and less than EPM order quantity for low-profit products when the market demand is subjected to normal distribution. It provides an alternative reason for the decision bias in the newsvendor model. (iv) When the newsvendor becomes more risk-averse and selects an optimal order quantity with a bigger confidence level to reduce the potential risks, he will expect a bigger opportunity loss and a smaller profit. Hence, the newsvendor must strike a balance between risk control and profit maximization (or opportunity loss minimization) in the determination of an order quantity. Thus, this study also provides insights into the risk management of the newsvendor model. This paper shows that the newsvendor's optimal order decision is related to the distribution of market demand when the newsvendor exhibits risk-averse preference. The theoretical and numerical results reveal that different market demands result in different optimal order decisions when the newsvendor exhibits riskaverse behavior. This leads to a practical question of how the risk-averse newsvendor should select the optimal order decision under different distributions of market demand. We believe that it is a more interesting issue to be discussed in the future.

Some extensions of this study are possible. For example, this paper does not consider the shortage cost for the lost sales (i.e., the excess demands that cannot be backlogged), while such a shortage cost ranges from the profit loss to some unspecific loss of unsatisfied customers' goodwill which always has an important influence on the benefit of the newsvendor. Therefore a possible extension is to integrate shortage cost into the definition of opportunity loss and then study the optimal ordering decisions of the newsvendor in a backorder setting.

\section{Appendix}

Proof of Theorem 1. For an order quantity $q$ and a realized market demand $D$, it follows from (3) that the opportunity loss of a newsvendor can be given as

$$
\begin{aligned}
& O(q)=(p-c) D-\left[p \min \{q, D\}-c q+r(q-D)^{+}\right. \\
& \left.\quad+w\left(p-c_{o}\right)(D-q)^{+}\right] .
\end{aligned}
$$

It follows from $\min \{q, D\}=q-(q-D)^{+}$and $(D-q)^{+}=$ $(D-q)+(q-D)^{+}$that

$$
\begin{aligned}
O(q)= & \left(p-c-w\left(p-c_{o}\right)\right)(D-q) \\
& +\left(p-r-w\left(p-c_{o}\right)\right)(q-D)^{+} .
\end{aligned}
$$

Then the newsvendor's expected opportunity loss $E[O(q)]$ can be given as

$$
\begin{aligned}
& E[O(q)] \\
& =\left(p-c-w\left(p-c_{o}\right)\right)(E(\xi)-q) \\
& \quad+\left(p-r-w\left(p-c_{o}\right)\right) \int_{0}^{q}(q-t) d F(t),
\end{aligned}
$$

which implies that

$$
\begin{aligned}
\frac{\partial E[O(q)]}{\partial q}= & -\left(p-c-w\left(p-c_{o}\right)\right) \\
& +\left(p-r-w\left(p-c_{o}\right)\right) F(q), \\
\frac{\partial^{2} E[O(q)]}{\partial q^{2}}= & \left(p-r-w\left(p-c_{o}\right)\right) f(q)>0 .
\end{aligned}
$$

It is concluded from (A.5) that $O(q)$ is convex in $q$. Then it follows from the first-order condition that $E[O(q)]$ attains the minimum in

$$
q^{*}=F^{-1}\left[\frac{p-c-w\left(p-c_{o}\right)}{p-r-w\left(p-c_{o}\right)}\right] .
$$

This completes the proof.

Proof of Theorem 2. For an order quantity $q$ and a realized market demand $D$, it follows from (A.2) that the newsvendor's opportunity loss is given as

$$
\begin{aligned}
O(q)= & \left(p-c-w\left(p-c_{o}\right)\right)(D-q) \\
& +\left(p-r-w\left(p-c_{o}\right)\right)(q-D)^{+} .
\end{aligned}
$$

We define an auxiliary function

$$
\begin{aligned}
& h(q, v)=v+\frac{1}{1-\alpha} E[O(q)-v]^{+} \\
& =v+\frac{1}{1-\alpha} \int_{0}^{+\infty}\left[\left(p-c-w\left(p-c_{o}\right)\right)(t-q)\right. \\
& \left.\quad+\left(p-r-w\left(p-c_{o}\right)\right)(q-t)^{+}-v\right]^{+} d F(t) \\
& =v+\frac{1}{1-\alpha} \int_{0}^{q}[(c-r)(q-t)-v]^{+} d F(t)+\frac{1}{1-\alpha} \\
& \quad \cdot \int_{q}^{+\infty}\left[\left(p-c-w\left(p-c_{o}\right)\right)(t-q)\right. \\
& \quad-v]^{+} d F(t) .
\end{aligned}
$$

It follows from the result in Rockafellar and Uryasev [34] that $h(q, v)$ is jointly convex in $(q, v)$. Further, it is concluded from the result in Section 2.2 that the optimal order quantity for a newsvendor to minimize CVaR of opportunity loss equals the optimal solution to the following problem:

$$
\min _{q \geq 0}\left[\min _{v \in R} h(q, v)\right]
$$


For any fixed $q$, we first discuss the optimal solution to problem $\min _{v \in R} h(q, v)$. We distinguish the following cases.

Case $1(v \geq(c-r) q)$. In this case, it follows from (A.8) that

$$
\begin{aligned}
& h(q, v)=v+\frac{1}{1-\alpha} \\
& \cdot \int_{q+v /\left(p-c-w\left(p-c_{o}\right)\right)}^{+\infty}\left[\left(p-c-w\left(p-c_{o}\right)\right)(t-q)\right. \\
& \quad-v] d F(t), \\
& \frac{\partial h(q, v)}{\partial v}=1-\frac{1}{1-\alpha}[1-F(q \\
& \left.\left.+\frac{v}{p-c-w\left(p-c_{o}\right)}\right)\right] .
\end{aligned}
$$

There exists sufficiently large $v^{\prime}$ such that $\left.(\partial h(q, v) / \partial v)\right|_{v=v^{\prime}} \geq$ 0 . If it satisfies $\left.(\partial h(q, v) / \partial v)\right|_{v=(c-r) q}=1-(1 /(1-\alpha))[1-F(q+$ $\left.\left.(c-r) q /\left(p-c-w\left(p-c_{o}\right)\right)\right)\right] \leq 0$, that is, $q \leq((p-c-w(p-$ $\left.\left.\left.c_{o}\right)\right) /\left(p-r-w\left(p-c_{o}\right)\right)\right) F^{-1}(\alpha)$, it follows from (A.11) that the optimal solution $v^{*}$ to problem $\min _{v \in R} \min h(q, v)$ solves

$$
1-\frac{1}{1-\alpha}\left[1-F\left(q+\frac{v^{*}}{p-c-w\left(p-c_{o}\right)}\right)\right]=0,
$$

which implies that

$$
v^{*}=\left(p-c-w\left(p-c_{o}\right)\right)\left(F^{-1}(\alpha)-q\right) .
$$

Case $2(0<v<(c-r) q)$. In this case, it follows from (A.8) that

$$
\begin{aligned}
& h(q, v)=v+\frac{1}{1-\alpha} \int_{0}^{q-v /(c-r)}[(c-r)(q-t) \\
& -v] d F(t)+\frac{1}{1-\alpha}
\end{aligned}
$$

$$
\begin{aligned}
& \cdot \int_{q+v /\left(p-c-w\left(p-c_{o}\right)\right)}^{+\infty}\left[\left(p-c-w\left(p-c_{o}\right)\right)(t-q)\right. \\
& -v] d F(t),
\end{aligned}
$$

$$
\begin{aligned}
& \frac{\partial h(q, v)}{\partial v}=1-\frac{1}{1-\alpha}\left[1+F\left(q-\frac{v}{c-r}\right)-F(q\right. \\
& \left.\left.+\frac{v}{p-c-w\left(p-c_{o}\right)}\right)\right] .
\end{aligned}
$$

It satisfies $\left.(\partial h(q, v) / \partial v)\right|_{v=0}=1-1 /(1-\alpha)<0$. If it satisfies $\left.(\partial h(q, v) / \partial v)\right|_{v=(c-r) q}=1-(1 /(1-\alpha))[1-F(q+(c-r) q /(p-$ $\left.\left.\left.c-w\left(p-c_{o}\right)\right)\right)\right] \geq 0$, that is, $q \geq\left(\left(p-c-w\left(p-c_{o}\right)\right) /(p-\right.$ $\left.\left.r-w\left(p-c_{o}\right)\right)\right) F^{-1}(\alpha)$, it follows from (A.15) that the optimal solution $v^{*}$ to problem $\min _{v \in R} h(q, v)$ solves

$$
\begin{aligned}
1 & -\frac{1}{1-\alpha}\left[1+F\left(q-\frac{v^{*}}{c-r}\right)\right. \\
& \left.-F\left(q+\frac{v^{*}}{p-c-w\left(p-c_{o}\right)}\right)\right]=0 .
\end{aligned}
$$

Case $3(v \leq 0)$. In this case, it follows from (A.8) that

$$
\begin{aligned}
& h(q, v)=v+\frac{1}{1-\alpha} \int_{0}^{q}[(c-r)(q-t)-v] d F(t) \\
& +\frac{1}{1-\alpha} \\
& \cdot \int_{q}^{+\infty}\left[\left(p-c-w\left(p-c_{o}\right)\right)(t-q)-v\right] d F(t), \\
& \frac{\partial h(q, v)}{\partial v}=1-\frac{1}{1-\alpha} \leq 0 .
\end{aligned}
$$

Due to the convexity of $h(q, \cdot)$, the minimum of $h(q, \cdot)$ cannot be attained in this case.

Based on the above analysis, it is clear that, for any fixed $q$, the optimal solution $v^{*}$ to problem $\min _{v \in R} h(q, v)$ is given by

$$
v^{*}= \begin{cases}\left(p-c-w\left(p-c_{o}\right)\right)\left(F^{-1}(\alpha)-q\right) & q \leq \frac{p-c-w\left(p-c_{o}\right)}{p-r-w\left(p-c_{o}\right)} F^{-1}(\alpha), \\ v^{\prime} & q \geq \frac{p-c-w\left(p-c_{o}\right)}{p-r-w\left(p-c_{o}\right)} F^{-1}(\alpha),\end{cases}
$$

where $v^{\prime}$ satisfies

$$
\begin{aligned}
1 & -\frac{1}{1-\alpha}\left[1+F\left(q-\frac{v^{\prime}}{c-r}\right)\right. \\
& \left.-F\left(q+\frac{v^{\prime}}{p-c-w\left(p-c_{o}\right)}\right)\right]=0 .
\end{aligned}
$$

To solve the problem $\min _{q \geq 0}\left[\min _{v \in R} h(q, v)\right]=$ $\min _{q \geq 0} h\left(q, v^{*}\right)$, we distinguish the following two cases.

(a) $q \leq\left(\left(p-c-w\left(p-c_{o}\right)\right) /\left(p-r-w\left(p-c_{o}\right)\right)\right) F^{-1}(\alpha)$. In this case, it follows from (A.18) that

$$
v^{*}=\left(p-c-w\left(p-c_{o}\right)\right)\left(F^{-1}(\alpha)-q\right) .
$$


Then it follows from (A.8) that

$$
\begin{aligned}
& h\left(q, v^{*}\right)=\left(p-c-w\left(p-c_{o}\right)\right)\left(F^{-1}(\alpha)-q\right) \\
& +\frac{1}{1-\alpha} \int_{F^{-1}(\alpha)}^{+\infty}\left(p-c-w\left(p-c_{o}\right)\right) \\
& \cdot\left(t-F^{-1}(\alpha)\right) d F(t) \\
& \frac{\partial h\left(q, v^{*}\right)}{\partial q}=-\left(p-c-w\left(p-c_{o}\right)\right)<0 .
\end{aligned}
$$
case.

Thus the minimum of $h\left(q, v^{*}\right)$ cannot be attained in this

(b) $q \geq\left(\left(p-c-w\left(p-c_{o}\right)\right) /\left(p-r-w\left(p-c_{o}\right)\right)\right) F^{-1}(\alpha)$. In this case, it follows from (A.18) and (A.19) that $v^{*}=v^{\prime}$ satisfies

$$
\begin{aligned}
1 & -\frac{1}{1-\alpha}\left[1+F\left(q-\frac{v^{\prime}}{c-r}\right)\right. \\
& \left.-F\left(q+\frac{v^{\prime}}{p-c-w\left(p-c_{o}\right)}\right)\right]=0 .
\end{aligned}
$$

Then it follows from (A.8) that

$$
\begin{aligned}
& h\left(q, v^{*}\right)=h\left(q, v^{\prime}\right)=v^{\prime}+\frac{1}{1-\alpha} \int_{0}^{q-v^{\prime} /(c-r)}[(c-r) \\
& \left.\cdot(q-t)-v^{\prime}\right] d F(t)+\frac{1}{1-\alpha} \\
& \cdot \int_{q+v^{\prime} /\left(p-c-w\left(p-c_{o}\right)\right)}^{+\infty}\left[\left(p-c-w\left(p-c_{o}\right)\right)(t-q)\right. \\
& \left.-v^{\prime}\right] d F(t), \\
& \frac{\partial h\left(q, v^{\prime}\right)}{\partial q}=\frac{1}{1-\alpha}\left[(c-r) F\left(q-\frac{v^{\prime}}{c-r}\right)-(p\right. \\
& \left.-c-w\left(p-c_{o}\right)\right)[1 \\
& \left.\left.-F\left(q+\frac{v^{\prime}}{p-c-w\left(p-c_{o}\right)}\right)\right]\right] .
\end{aligned}
$$

It follows from (A.24) and the first-order condition that the optimal solution $q^{\alpha}$ to problem $\min _{q \geq 0} h\left(q, v^{*}\right)=$ $\min _{q \geq 0} h\left(q, v^{\prime}\right)$ satisfies

$$
\begin{gathered}
(c-r) F\left(q^{\alpha}-\frac{v^{\prime}}{c-r}\right)-\left(p-c-w\left(p-c_{o}\right)\right) \\
\cdot\left[1-F\left(q^{\alpha}+\frac{v^{\prime}}{p-c-w\left(p-c_{o}\right)}\right)\right]=0 .
\end{gathered}
$$

It follows from (A.19) and (A.25) that the optimal solution $q^{\alpha}$ to problem $\min _{q \geq 0} h\left(q, v^{*}\right)$ is given as

$$
\begin{aligned}
q^{\alpha} & =\left((c-r) F^{-1}\left[\frac{(1-\alpha)\left(p-c-w\left(p-c_{o}\right)\right)}{p-r-w\left(p-c_{o}\right)}\right]\right. \\
& +\left(p-c-w\left(p-c_{o}\right)\right) \\
& \left.\cdot F^{-1}\left[\frac{(1-\alpha)\left(p-c-w\left(p-c_{o}\right)\right)}{p-r-w\left(p-c_{o}\right)}+\alpha\right]\right)(p \\
& \left.-r-w\left(p-c_{o}\right)\right)^{-1} .
\end{aligned}
$$

This completes the proof.

Proof of Theorem 5. For an order quantity $q$ and a realized market demand $D$, it follows from (1) that a newsvendor's realized profit is given as

$$
\begin{aligned}
P(q)= & p \min \{q, D\}-c q+r(q-D)^{+} \\
& +w\left(p-c_{o}\right)(D-q)^{+} .
\end{aligned}
$$

It follows from $\min \{q, D\}=q-(q-D)^{+}$and $(D-q)^{+}=$ $(D-q)+(q-D)^{+}$that

$$
\begin{aligned}
P(q)= & \left(p-c-w\left(p-c_{o}\right)\right) q+w\left(p-c_{o}\right) D \\
& -\left(p-r-w\left(p-c_{o}\right)\right)(q-D)^{+} .
\end{aligned}
$$

Then the newsvendor's expected profit of $E[P(q)]$ can be given as

$$
\begin{aligned}
E[ & P(q)] \\
= & \left(p-c-w\left(p-c_{o}\right)\right) q+w\left(p-c_{o}\right) E(\xi) \\
& \quad-\left(p-r-w\left(p-c_{o}\right)\right) \int_{0}^{q}(q-t) d F(t) .
\end{aligned}
$$

It follows that

$$
\begin{gathered}
\frac{\partial E[P(q)]}{\partial \alpha}=\left[\left(p-c-w\left(p-c_{o}\right)\right)\right. \\
\left.-\left(p-r-w\left(p-c_{o}\right)\right) F(q)\right] \frac{\partial q}{\partial \alpha} \\
\frac{\partial E\left[P\left(q^{\alpha}\right)\right]}{\partial \alpha}=\left[\left(p-c-w\left(p-c_{o}\right)\right)\right. \\
\left.-\left(p-r-w\left(p-c_{o}\right)\right) F\left(q^{\alpha}\right)\right] \frac{\partial q^{\alpha}}{\partial \alpha} .
\end{gathered}
$$

By Remark 3, if the optimal order quantity $q^{\alpha}$ is increasing in the confidence level $\alpha$, we have $\partial q^{\alpha} / \partial \alpha \geq 0$ and $q^{\alpha} \geq q^{*}$. Then it follows from $q^{*}=F^{-1}\left[\left(p-c-w\left(p-c_{o}\right)\right) /\left(p-r-w\left(p-c_{o}\right)\right)\right]$ that

$$
\begin{gathered}
\left(p-c-w\left(p-c_{o}\right)\right)-\left(p-r-w\left(p-c_{o}\right)\right) F\left(q^{\alpha}\right) \\
\leq\left(p-c-w\left(p-c_{o}\right)\right) \\
\quad-\left(p-r-w\left(p-c_{o}\right)\right) F\left(q^{*}\right)=0 .
\end{gathered}
$$


It follows from (A.31), (A.32), and $\partial q^{\alpha} / \partial \alpha \geq 0$ that

$$
\frac{\partial E\left[P\left(q^{\alpha}\right)\right]}{\partial \alpha} \leq 0,
$$

which proves that $E\left[P\left(q^{\alpha}\right)\right]$ is decreasing in the confidence level $\alpha$. Otherwise, the optimal order quantity $q^{\alpha}$ is decreasing in the confidence level $\alpha$; we have $\partial q^{\alpha} / \partial \alpha \leq 0$ and $q^{\alpha} \leq q^{*}$. Then it follows from $q^{*}=F^{-1}\left[\left(p-c-w\left(p-c_{o}\right)\right) /(p-r-\right.$ $\left.\left.w\left(p-c_{o}\right)\right)\right]$ that

$$
\begin{aligned}
& \left(p-c-w\left(p-c_{o}\right)\right)-\left(p-r-w\left(p-c_{o}\right)\right) F\left(q^{\alpha}\right) \\
& \geq\left(p-c-w\left(p-c_{o}\right)\right) \\
& \quad-\left(p-r-w\left(p-c_{o}\right)\right) F\left(q^{*}\right)=0 .
\end{aligned}
$$

It follows from (A.31), (A.34), and $\partial q^{\alpha} / \partial \alpha \leq 0$ that

$$
\frac{\partial E\left[P\left(q^{\alpha}\right)\right]}{\partial \alpha} \leq 0,
$$

which proves that $E\left[P\left(q^{\alpha}\right)\right]$ is decreasing in the confidence level $\alpha$. This completes the proof.

Proof of Theorem 6. The proof is similar to that of Theorem 5, which is omitted.

\section{Conflicts of Interest}

The authors declare that there are no conflicts of interest regarding the publication of this paper.

\section{Acknowledgments}

This work was supported by the Research Committee of the Hong Kong Polytechnic University, the China Postdoctoral Science Foundation (Grant 2016M590697), and the Science Foundation of Binzhou University (Grant 2015Y10).

\section{Endnotes}

1. In Schweitzer and Cachon [19], these authors define a product as a high-profit product when $\phi \geq 1 / 2$, where $\phi$ is the critical fractile. Otherwise, the product is defined as a low-profit product. In our paper, it follows from Theorem 1 that the critical fractile is given as $(p-c-$ $\left.w\left(p-c_{o}\right)\right) /\left(p-r-w\left(p-c_{o}\right)\right) . \phi=\left(p-c-w\left(p-c_{o}\right)\right) /(p-$ $\left.r-w\left(p-c_{o}\right)\right) \geq 1 / 2$ is equal to

$$
p-c-w\left(p-c_{o}\right) \geq c-r .
$$

Thus high profit means that understock loss is bigger than overstock loss and low profit means that understock loss is smaller than overstock loss.

\section{References}

[1] M. Khouja, "The single-period (news-vendor) problem: literature review and suggestions for future research," Omega, vol. 27, no. 5, pp. 537-553, 1999.
[2] Y. Qin, R. Wang, A. J. Vakharia, Y. Chen, and M. M. H. Seref, "The newsvendor problem: review and directions for future research," European Journal of Operational Research, vol. 213, no. 2, pp. 361-374, 2011.

[3] E. Arkkan and J. Fichtinger, "The risk-averse newsvendor problem under spectral risk measures: a classification with extensions," European Journal of Operational Research, vol. 256, no. 1, pp. 116-125, 2017.

[4] D. Corsten and T. Gruen, "Stock-outs cause walkouts," Harvard Business Review, vol. 86, no. 5, pp. 26-28, 2004.

[5] D. C. Montgomery, M. S. Bazaraa, and A. K. Keswani, "Inventory models with a mixture of backorders and lost sales," Naval Research Logistics, vol. 20, no. 2, pp. 255-263, 1973.

[6] Z. K. Weng, "Coordinating order quantities between the manufacturer and the buyer: a generalized newsvendor model," European Journal of Operational Research, vol. 156, no. 1, pp. 148-161, 2004.

[7] L. A. San José, J. Sicilia, and J. García-Laguna, "Analysis of an inventory system with exponential partial backordering," International Journal of Production Economics, vol. 100, no. 1, pp. 76-86, 2006.

[8] E. J. Lodree Jr., "Advanced supply chain planning with mixtures of backorders, lost sales, and lost contract," European Journal of Operational Research, vol. 181, no. 1, pp. 168-183, 2007.

[9] J. E. J. Lodree, Y. Kim, and W. Jang, “Time and quantity dependent waiting costs in a newsvendor problem with backlogged shortages," Mathematical and Computer Modelling, vol. 47, no. 1-2, pp. 60-71, 2008.

[10] Y.-W. Zhou and S.-D. Wang, "Manufacturer-buyer coordination for newsvendor-type-products with two ordering opportunities and partial backorders," European Journal of Operational Research, vol. 198, no. 3, pp. 958-974, 2009.

[11] V. Pando, L. A. San-José, J. García-Laguna, and J. Sicilia, "A newsboy problem with an emergency order under a general backorder rate function," Omega (United Kingdom), vol. 41, no. 6, pp. 1020-1028, 2013.

[12] J. Chen, S. Huang, R. Hassin, and N. Zhang, "Two backorder compensation mechanisms in inventory systems with impatient customers," Production and Operations Management, vol. 24, no. 10, pp. 1640-1656, 2015.

[13] S. Liu, M. Song, K. C. Tan, and C. Zhang, "Multi-class dynamic inventory rationing with stochastic demands and backordering," European Journal of Operational Research, vol. 244, no. 1, pp. 153-163, 2015.

[14] L.-F. Hsu and J.-T. Hsu, "Economic production quantity (EPQ) models under an imperfect production process with shortages backordered," International Journal of Systems Science. Principles and Applications of Systems and Integration, vol. 47, no. 4, pp. 852-867, 2016.

[15] M. Braglia, D. Castellano, and M. Gallo, "Approximated closedform minimum-cost solution to the $(r, q)$ policy with complete backordering and further developments," Applied Mathematical Modelling. Simulation and Computation for Engineering and Environmental Systems, vol. 40, no. 19-20, pp. 8406-8423, 2016.

[16] S. Khalilpourazari, S. H. Pasandideh, and S. T. Niaki, “Optimization of multi-product economic production quantity model with partial backordering and physical constraints: SQP, SFS, SA, and WCA," Applied Soft Computing, vol. 49, pp. 770-791, 2016.

[17] A. A. Taleizadeh, H. R. Zarei, and B. R. Sarker, "An optimal control of inventory under probablistic replenishment intervals 
and known price increase," European Journal of Operational Research, vol. 257, no. 3, pp. 777-791, 2017.

[18] S. Khalilpourazari and S. H. R. Pasandideh, "Multi-item EOQ model with nonlinear unit holding cost and partial backordering: moth-flame optimization algorithm," Journal of Industrial and Production Engineering, vol. 34, no. 1, pp. 42-51, 2017.

[19] M. E. Schweitzer and G. P. Cachon, "Decision bias in the newsvendor problem with a known demand distribution: experimental evidence," Management Science, vol. 46, no. 3, pp. 404-420, 2000.

[20] C. X. Wang and S. Webster, "The loss-averse newsvendor problem," Omega, vol. 37, no. 1, pp. 93-105, 2009.

[21] G. E. Bolton and E. Katok, "Learning by doing in the newsvendor problem: a laboratory investigation of the role of experience and feedback," Manufacturing and Service Operations Management, vol. 10, no. 3, pp. 519-538, 2008.

[22] M. Kremer, S. Minner, and L. N. Van Wassenhove, "Do random errors explain newsvendor behavior?" Manufacturing and Service Operations Management, vol. 12, no. 4, pp. 673-681, 2010.

[23] T.-H. Ho, N. Lim, and T. H. Cui, "Reference dependence in multilocation newsvendor models: a structural analysis," Management Science, vol. 56, no. 11, pp. 1891-1910, 2010.

[24] M. Nagarajan and S. Shechter, "Prospect theory and the newsvendor problem," Management Science, vol. 60, no. 4, pp. 1057-1062, 2014.

[25] X. Long and J. Nasiry, "Prospect theory explains newsvendor behavior: the role of reference points," Management Science, vol. 61, no. 12, pp. 3009-3012, 2015.

[26] N. Lau, S. Hasija, and J. N. Bearden, "Newsvendor pull-to-center reconsidered," Decision Support Systems, vol. 58, no. 1, pp. 6873, 2014.

[27] X.-B. Zhao and W. Geng, "A note on "Prospect theory and the newsvendor problem"," Journal of the Operations Research Society of China, vol. 3, no. 1, pp. 89-94, 2015.

[28] Y. Zhao and X. Zhao, "How a competing environment influences newsvendor ordering decisions," International Journal of Production Research, vol. 54, no. 1, pp. 204-214, 2016.

[29] Y. Lan, H. Gao, M. O. Ball, and I. Karaesmen, "Revenue management with limited demand information," Management Science, vol. 54, no. 9, pp. 1594-1609, 2008.

[30] T. S. Ng, "Robust regret for uncertain linear programs with application to co-production models," European Journal of Operational Research, vol. 227, no. 3, pp. 483-493, 2013.

[31] J. Ruan and Y. Shi, "Monitoring and assessing fruit freshness in IOT-based e-commerce delivery using scenario analysis and interval number approaches," Information Sciences, vol. 373, pp. 557-570, 2016.

[32] J. H. Ruan, X. P. Wang, F. T. S. Chan, and Y. Shi, "Optimizing the intermodal transportation of emergency medical supplies using balanced fuzzy clustering," International Journal of Production Research, vol. 54, no. 14, pp. 4368-4386, 2016.

[33] R. T. Rockafellar and S. Uryasev, "Optimization of conditional value-at-risk," The Journal of Risk, vol. 2, pp. 21-41, 2000.

[34] R. T. Rockafellar and S. Uryasev, "Conditional value-at-risk for general loss distributions," Journal of Banking and Finance, vol. 26, no. 7, pp. 1443-1471, 2002.

[35] X. Chen, M. Sim, D. Simichi-Levi, and P. Sun, Risk Aversion in Inventory Management, MIT, Cambridge, UK, 2003.

[36] J.-Y. Gotoh and Y. Takano, "Newsvendor solutions via conditional value-at-risk minimization," European Journal of Operational Research, vol. 179, no. 1, pp. 80-96, 2007.
[37] Y. Chen, M. Xu, and Z. G. Zhang, "A risk-averse newsvendor model under the CVaR criterion," Operations Research, vol. 57, no. 4, pp. 1040-1044, 2009.

[38] S. Eskandarzadeh and K. Eshghi, "Decision tree analysis for a risk averse decision maker: CVaR criterion," European Journal of Operational Research, vol. 231, no. 1, pp. 131-140, 2013.

[39] M. Wu, S. X. Zhu, and R. H. Teunter, "A risk-averse competitive newsvendor problem under the CVaR criterion," International Journal of Production Economics, vol. 156, pp. 13-23, 2014.

[40] T. C. Y. Chan, H. Mahmoudzadeh, and T. G. Purdie, "A robustCVaR optimization approach with application to breast cancer therapy," European Journal of Operational Research, vol. 238, no. 3, pp. 876-885, 2014.

[41] X. Xu, Z. Meng, R. Shen, M. Jiang, and P. Ji, "Optimal decisions for the loss-averse newsvendor problem under CVaR," International Journal of Production Economics, vol. 164, pp. 146159, 2015.

[42] X. Xu, Z. Meng, P. Ji, C. Dang, and H. Wang, "On the newsvendor model with conditional Value-at-Risk of opportunity loss," International Journal of Production Research, vol. 54, no. 8, pp. 2449-2458, 2016.

[43] L. Eeckhoudt, C. Gollier, and H. Schlesinger, "The risk-averse (and prudent) newsboy," Management Science, vol. 41, no. 5, pp. 786-794, 1995. 


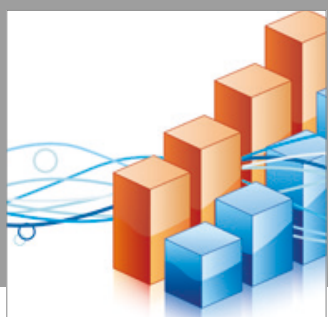

Advances in

Operations Research

vatersals

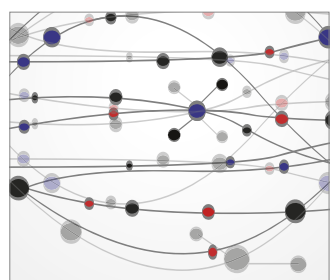

\section{The Scientific} World Journal
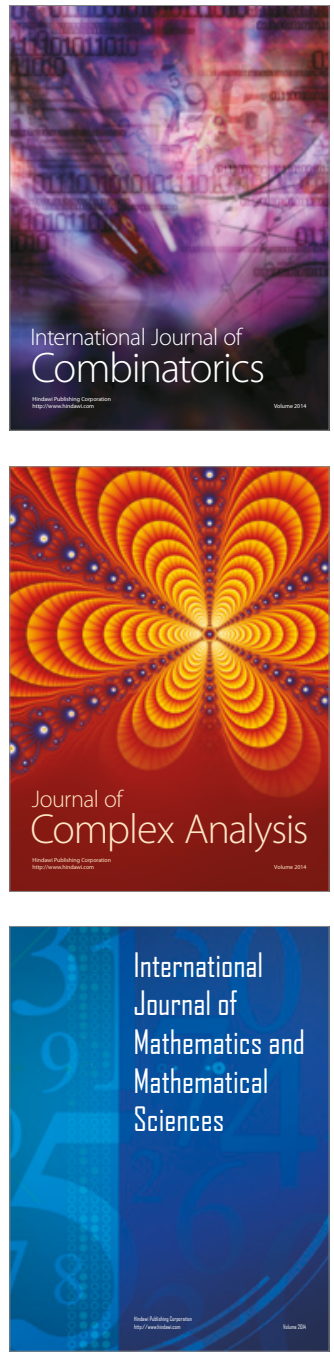
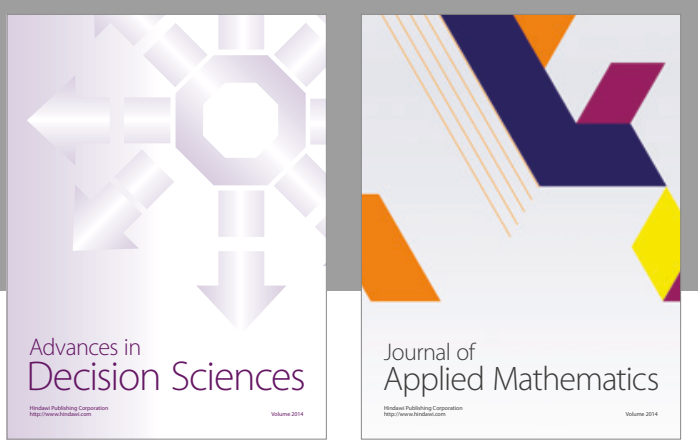

Algebra

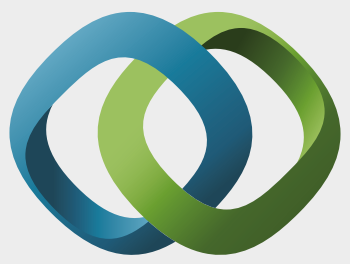

\section{Hindawi}

Submit your manuscripts at

https://www.hindawi.com
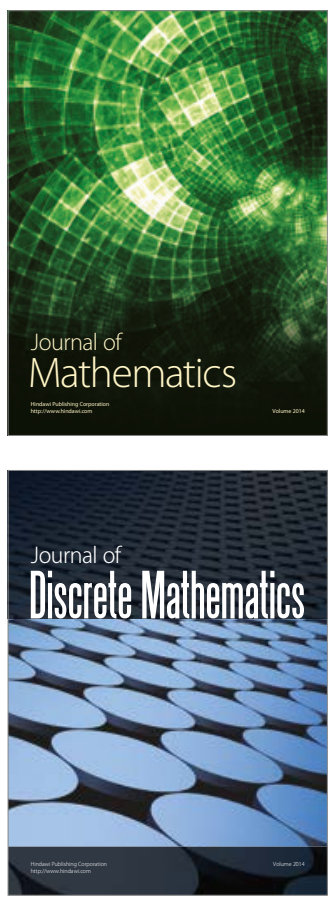

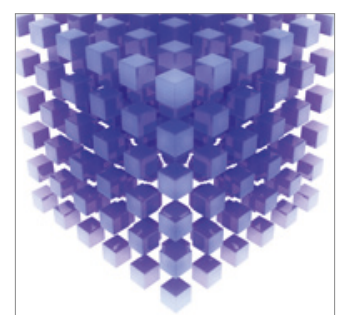

Mathematical Problems in Engineering
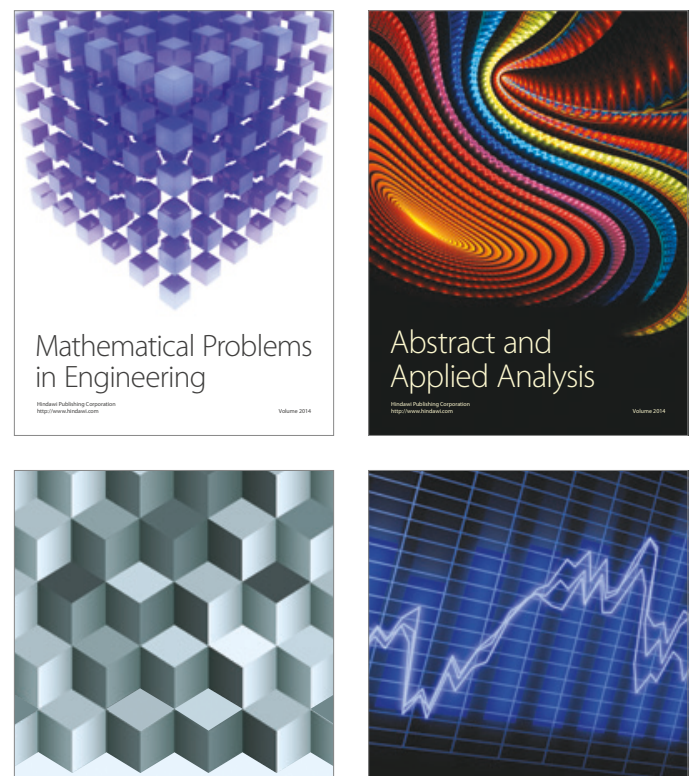

Journal of

Function Spaces

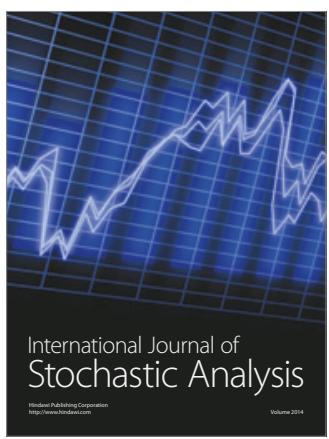

Probability and Statistics
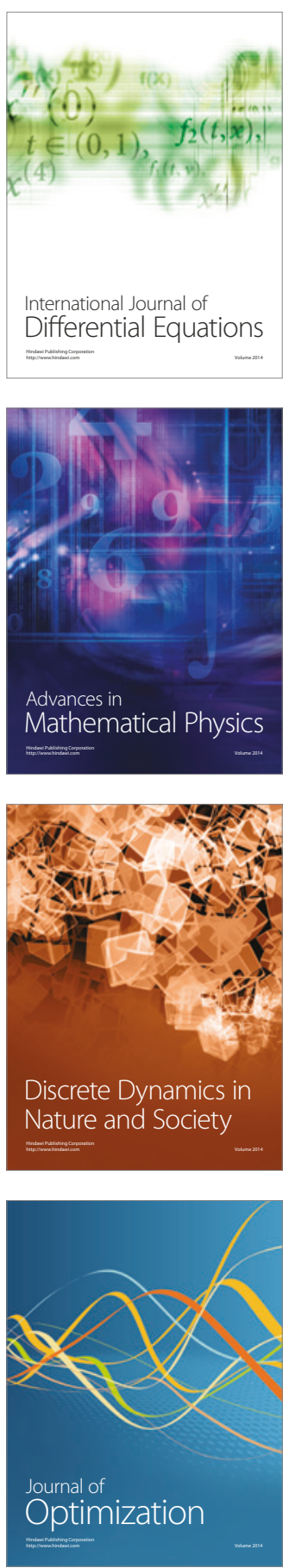